\section{SPACEMAKER: A program to increase Apple disk capacity from 31 to 33 tracks}

\section{ELIOT SHIMOFF \\ University of Maryland Baltimore County, Baltimore, Maryland}

SPACEMAKER is an Apple II DOS 3.3. disk utility program that provides a quick method of determining the space remaining on a disk, and frees two additional tracks (a total of 32 sectors) of disk space for programs or text files.

Every disk initialized by Apple's Disk Operating System (DOS 3.3) has 35 concentric tracks (much like grooves of a phonograph record), each divided into 32 sectors. Each sector can store 256 bytes (characters). Not all of these tracks are available for data or program files; Track 17, the center track, is reserved by DOS for the catalog and a table of contents (described below), while DOS itself is stored on tracks 0,1 , and 2 .

Having the "DOS image" on the first three tracks of a disk is often a convenience; when the disk is booted, DOS automatically is available. But disks containing only text files for access by word-processing programs, database management packages, spreadsheets, or data analysis routines are never directly booted, and the DOS stored on Tracks 0,1 , and 2 is never used; files on such disks are read into memory by other programs. Although it is impossible to store files on Track 0 without rewriting parts of DOS itself, it is relatively easy to gain access to Tracks 1 and 2 and to increase effective disk capacity from 31 tracks to 33 tracks. (As noted above, the disk is initialized with 35 tracks, but Track 0 is inaccessible, and Track 17 is reserved for the directory, leaving 33 tracks available.)

In order to clear Tracks 1 and 2 for file storage, a user must modify the Volume Table of Contents (VTOC), a special file stored in Sector 0 of Track 17. The VTOC is not accessible through DOS itself, but can be modified through a DOS subroutine called Read or Write a Track and Sector (RWTS). The RWTS routine allows the user to read from or write to any sector or any track. RWTS looks for an Input/Output Control Block (IOB) and Device Characteristic Table (DCT) to determine which slot, drive, track, and sector to operate on, and what operations to perform (i.e., read, write, initialize). (Details of the IOB and DCT are provided in the Apple II DOS Manual, pp. 94-98). The SPACEMAKER program (see Appendix) uses GET.VTOC to create the IOB and DCT; the 19456 CALL, at the end of GET.VTOC, is to RWTS, which examines the IOB and DCT (created by the POKEs), and places the VTOC into memory.

Address correspondence to: Eliot Shimoff, Department of Psychology, University of Maryland Baltimore County, Baltimore, MD 21228.
The VTOC includes a "Track Bit Map," in which every sector of every track is represented by a single bit set at " 1 " if the sector is unused or " 0 " if used. If the user requests "Space on Disk" from the MENU, DECODE examines each of the memory locations of the Track Bit Map, using BITCOUNT to count the number of bits set to " 1 .",

If the user requests the program to clear Tracks 1 and 2 for files (CLEAR.DOS), the program first checks the Track Bit Map for Track 0 (20536 PEEK $0=$ IF). Tracks 1 and 2 will be cleared only if the Track Bit Map indicates that Track 0 is "in use" (i.e., is set to 0 ); if Track 0 is marked as clear, Tracks 1 and 2 have already been freed for file storage. If the tracks have not been freed yet, the program will set the Track Bit Map to show the Tracks 0, 1 , and 2 as available (the DO loop in CLEAR.DOS); the IOB is modified to write to the disk by POKEing a 2 into location 19478; RWTS is called to store the VTOC back on the disk by CALLing 19456; and the IOB is reset to read the VTOC by POKEing 1 into location 19478.

Once files have been written on Tracks 1 and 2, the disk cannot be booted; attempts at booting (e.g., by typing "PR\#6") will fail, but there will be no damage to the disk, the drive, or the computer.

\section{AVAILABILITY}

The Appendix shows a full listing of the FORTH (specifically, transFORTH Ib) program. SPACEMAKER is also available as a BASIC program; the listing is available from the author.

\section{REFERENCE}

Apple II DOS Manual. (1981). Cupertino, CA: Apple Computer Co.

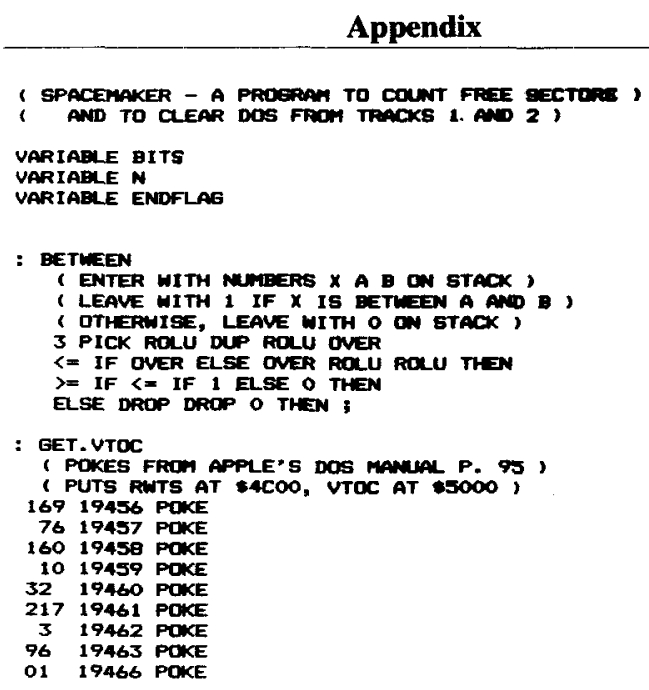




$\begin{array}{rll}96 & 19467 & \text { POKE } \\ 01 & 19468 & \text { POKE } \\ 00 & 19469 & \text { POKE } \\ 17 & 19470 & \text { POKE } \\ 0 & 19471 & \text { POKE } \\ 32 & 19472 & \text { POKE } \\ 76 & 19473 & \text { POKE } \\ 0 & 19474 & \text { POKE } \\ 80 & 19475 & \text { POKE } \\ 0 & 19476 & \text { POKE } \\ 0 & 19477 & \text { POKE } \\ 01 & 19478 & \text { POKE } \\ 00 & 19479 & \text { POKE } \\ 00 & 19480 & \text { POKE } \\ 96 & 19481 & \text { POKE } \\ 01 & 19492 & \text { POKE } \\ 0 & 19489 & \text { POKE } \\ 01 & 19489 & \text { POKE } \\ 239 & 19490 & \text { POKE } \\ 216 & 19491 & \text { POKE }\end{array}$

21619491 POKE

1945

\section{= BITCOUNT}

2 COUNTS BITS SET

( ENTER WITH MUMBER TO BE COUNTED WAITING )

$\rightarrow N$ ( NUMBER TO BE TESTED)

$N 128>=$ IF BITS $1+\rightarrow$ BITS $N 128-\rightarrow N$ THEN

$N 64>=$ IF BITS $1+->$ BITS N $64-\rightarrow N$ THEN

$N 32>=$ IF BITS $1+\rightarrow$ BITS $N 32-\rightarrow$ NTHEN

$N 16>=$ IF BITS $1+\rightarrow$ BITS N $16-\rightarrow N$ THEN

$N A>=$ IF BITS $1+->$ GITS $N B-\rightarrow N$ THEN

$N 4>=$ IF BITS $1+\rightarrow$ BITS $N 4-\rightarrow N$ THEN

N $2>$ IF BITS $1+\rightarrow$ BITS N $2-\rightarrow N$ THEN

$N 1 \lambda=$ IF BITS $1+\rightarrow$ BITS THEN

: DECODE

( DECODE TRACK BIT MAP AT $\$ 5000$

BET. VTOC

2067520540 DO ( GET TRACK MAPS FOR ALL BUT TRACK O) I PEEK BITCOUNT

I 1 + PEEK BITCOUNT

+LOOP

( COUNT BITS SET FOR EACH TRACK )

PRINT" "SPACE ON DISK: " IMUERSE BITS - NORMAL CR CR PRINT " PRESS RETURN TO CONTINUE " PAD READLN

:

- SHOW maP HOME

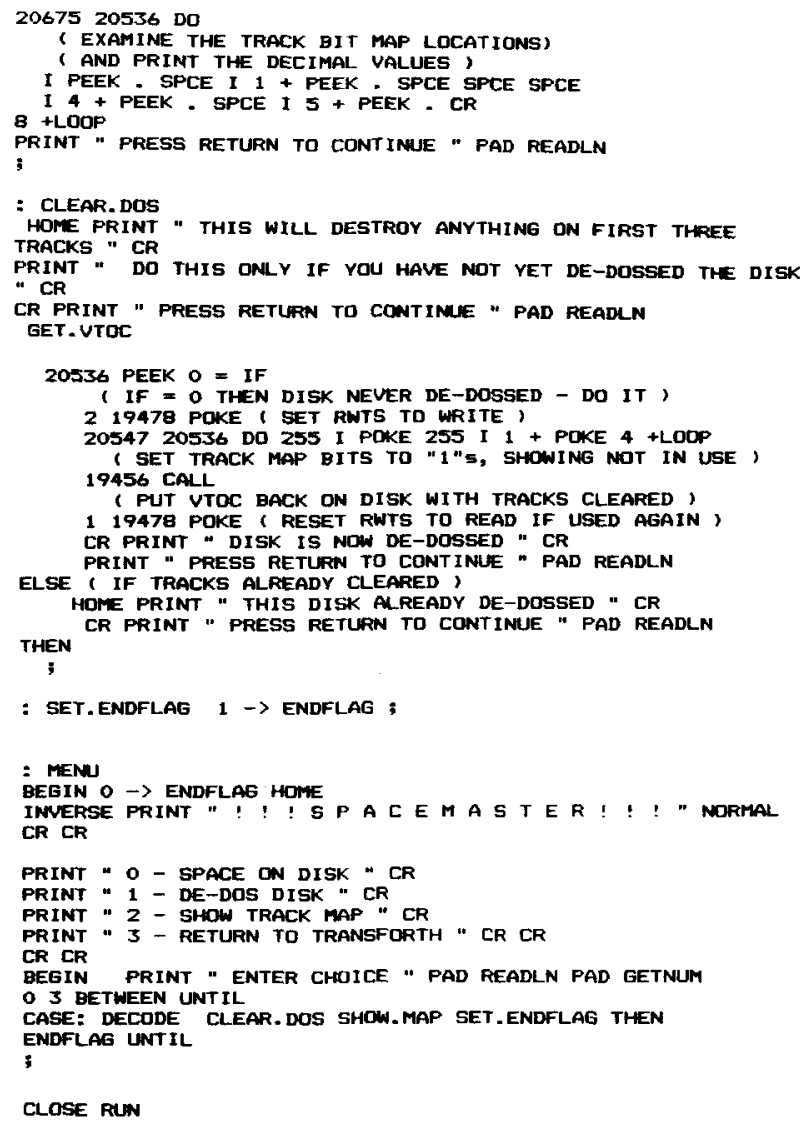

(Manuscript received March 12, 1985;) revision accepted for publication May 8, 1985.) 